\title{
RESPONSES OF PRIMARY AUDITORY NEURONS TO ELECTRO-MAGNETIC DRIVING OF THE EAR DRUM
}

\author{
Takeshi WATANABE \\ Laboratory for the Deaf, Tokyo Medical and Dental University
}

Considering the problem of the relation between basilar membrane displacement and the initiation of nerve impulses at the nerve endings in the cochlea, DAVIS, FERnANDEZ, and McAulifFE ${ }^{3)}$ have suggested that motion of the basilar membrane in only one direction (i. e., corresponding to outward displacement of the stapes) is capable of producing neural excitation. This would correspond to movement of the basilar membrane toward the scala vestibuli. In order to test this possibility the present study has employed a step-function type of stimulus in either a positive or a negative direction through conventional electro-magnetic driving of the ear drum.

Since the nerve impulses are transmitted along their axons without any synaptic interaction between adjacent neurons, it should be possible to gain information concerning the physiology of the cochlea indirectly by studying the response pattern of single primary auditory neurons. This paper reports qualitative results recorded in this manner.

\section{METHODS}

Seven adult cats weighing $2.5 \mathrm{Kg}$ or more used as subjects, which were administered Dial with urethane $(60 \mathrm{mg} / \mathrm{Kg})$ as an anesthetic.

The internal auditory meatus was exposed using an intracranial approach. Clear exposure of the internal auditory meatus was accomplished by lifting the cerebellum after partial removal of the tentorium cerebri. The external auditory meatus was removed at its attachment of the skull.

The animals were then mounted on the Horsley-Clarke stereo-taxic instrument. In this case, the ear bars were fixed in small holes drilled bilaterally in the skull. The magnetic sheet was carefully pasted on the ear drum and the magnetic coil placed very close to the ear drum.

Stimulating System. A) Sounds: Both clicks and tone bursts were used as sound stimuli in a free field. Clicks were produced by $100 \mu \mathrm{sec}$ square-wave pulses from

Received for publication October 17, 1964

渡辺武 
Tektronix 161 pulse generators. Tone bursts were obtained by an oscillator and controlled by an electronic switch (Grason Stadler Model 829B). B) Electro-magnetic driving of the ear drum: Powdered permanent magnets were pasted on the magnetic tape of about $2 \mathrm{~mm}^{2}$ with the photopaste. Such small pieces of magnetic sheets were polarized by placing them on either pole of a powerful magnet. The weight of the magnetic sheets in this experiment ranged from $1.8 \mathrm{mg}$ to $5.4 \mathrm{mg}$. The driving coil was made of a mechanical relay having very little residual magnetism. The magnetic sheet was pasted on the ear drum around the Pars tensa with Alon Alpha (a type of tissue adhesive provided by Sankyo-Company, Tokyo). In all these experiments, an attraction of the ear drum occurred when a positive pulse was applied to the coil and repulsion occurred when the driving polarity was reversed. The stimulus strength mentioned refers to the calibrated dial voltage of the stimulator. The actual displacement of the ear drum was not measured in this study.

Recording System. A hyperfine microelectrode was inserted into the auditory nerve bundle from its posterior surface in an antero-ventral direction. The electrodes used for recording single fiber responses were glass capillaries filled with a 3 molar $\mathrm{KCl}$ solution with a resistance between 40 to $90 \mathrm{M} \Omega$. The action potentials were fed into a cathode follower head amplifier, then to a Tektronix 502 oscilloscope which was equipped a high gain amplifier.

\section{RESULTS}

Influences of the loading upon cochlear microphonics. After pasting the small piece of magnetic sheet on the ear drum the sensitivity of the cochlear microphonics is changed due to the increased mass. FIG. 1 shows the change of

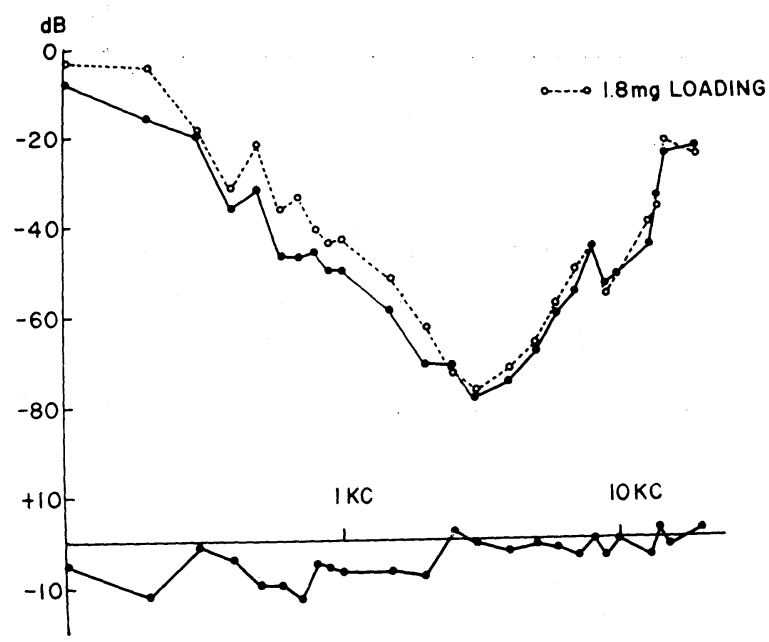

FIG. 1. Audibility curves determined by recording cochlear microphonics before (solid line) and after (dotted line) loading of $1.8 \mathrm{mg}$ magnetic sheet on the ear drum. The lower figure represents the difference between the two cases. 
an audibility curve before and after loading of the $1.8 \mathrm{mg}$ magnetic sheet. There is a small elevation of threshold below $2 \mathrm{Kc}$, but the threshold does not change as much over the higher frequency range. Similar trials were done with a load up to $5.4 \mathrm{mg}$, and the same tendency noticed. Therefore, it is possible to determine the characteristic frequency of the primary auditory neurons accurately even though the loading amounted to $5.4 \mathrm{mg}$.

Unit responses to a step-function type of stimulus. The discharge pattern of a single primary auditory neurons in response to a step-function type of magnetic driving of the ear drum is shown in FIG. 2. The near-threshold response consists of three spikes which appear about $6.25 \mathrm{msec}$ after the application of either a positive or a negative step function stimulus. As the stimulus strength is increased the number of spikes within the burst increases. In this particular case the unit has a characteristic frequency of $400 \mathrm{cps}$. Other units with characteristic frequencies of $700 \mathrm{cps}, 900 \mathrm{cps}$ and $9500 \mathrm{cps}$ are shown in FIgs. 3A, 3B and 3C, respectively. As seen in FIG. 3A, on and off responses have been recorded using two durations of the stimulus. In the units with high characteristic frequencies the number of impulses does not change as the stimulus strength is increased. FIG. 3C illustrates an example where only

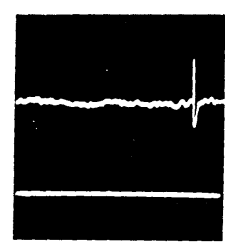

spontaneous
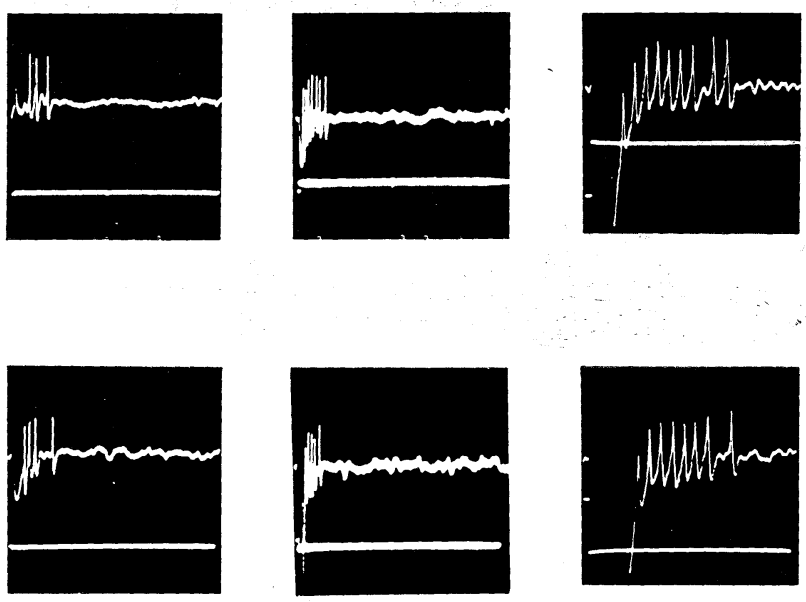

$\overline{50 \mathrm{~ms}}$

upper : attraction

lower: repulsion

FIG. 2. Response pattern of a single primary auditory neuron with a characteristic frequency of $400 \mathrm{cps}$ in response to step-function magnetic driving of the ear drum. Left figure: Spontaneous discharge. Top row: Attraction. Bottom row: Repulsion. From left to right the driving voltage was increased. In each figure the upper beam shows unit responses and the lower beam the monitored driving voltage of the magnetic coil. 


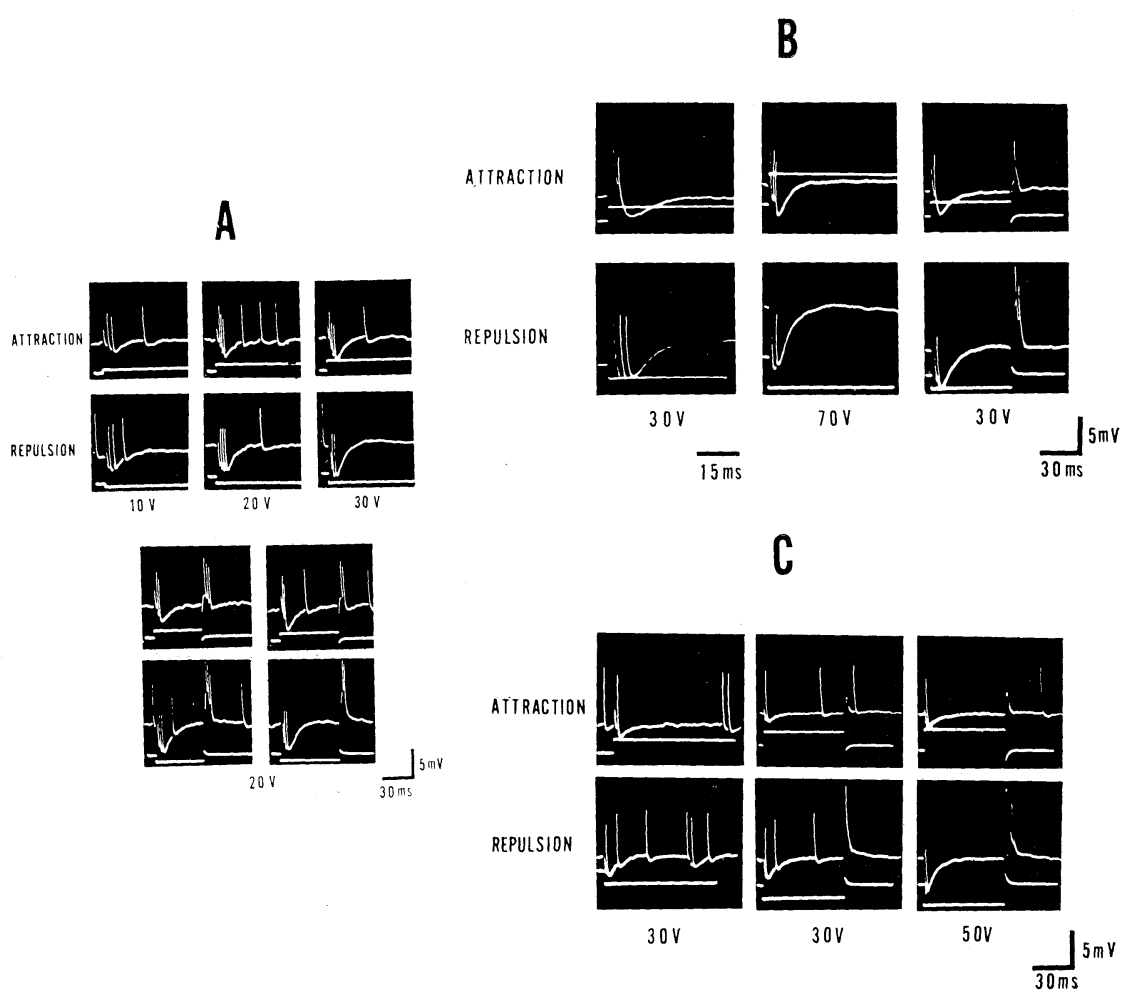

FIG. 3. A : Unit responses to step-function electro-magnetic driving of the ear drum. This unit has a characteristic frequency of $700 \mathrm{cps}$. Top figure: Responses to an attraction and repulsion of the ear drum at three different intensity levels as indicated. Bottom figure: On and off responses to either an attraction or repulsion of the ear drum using two different stimulus durations but the same intensity level. B: Unit has a characteristic frequency of $900 \mathrm{cps}$. C: Unit has a characteristic frequency of $9500 \mathrm{cps}$.

a single spike could be elicited. Repetitive firing in units with a low characteristic frequency (below $1 \mathrm{kc}$ ) generally occurs in response to magnetic driving of the ear drum.

Latency of responses. Measurements were made of the latency of responses to magnetic driving of the ear drum. The dotted pattern of the responses to both attraction (outward deflection of the ear drum) and repulsion (inward deflection of the ear drum) of the tympanic membrane is shown in Fig. 4. This unit has a characteristic frequency of $700 \mathrm{cps}$ at $-80 \mathrm{~dB}$. In this figure the solid circles indicate responses to attraction of the ear drum, and the empty circles show the responses to repulsion of the ear drum at three different intensity levels. In this unit repetitive firing was observed with a small fluctuation of the latency. When the stimulus intensity was increased 


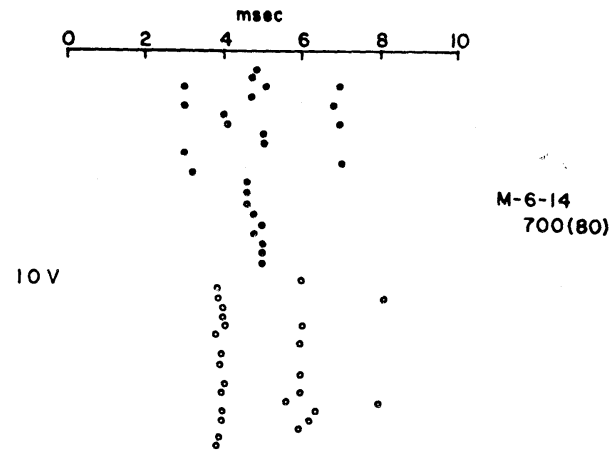

$20 \mathrm{~V}$

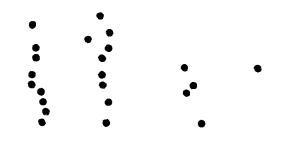

$30 \mathrm{~V}$
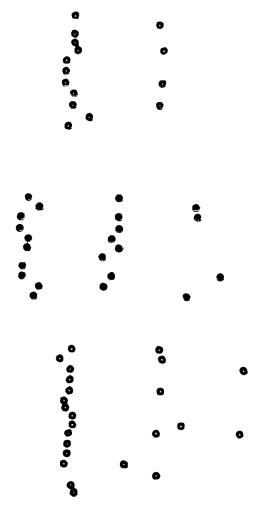

FIG. 4. The dotted pattern of the responses to both attraction (solid circles) and repulsion (open circles) of the ear drum at three different intensity levels. Characteristic frequency of this unit is $700 \mathrm{cps}$ at -80 $\mathrm{dB}$.

the first series of responses synchronized fairly well and the latency gradually decreased. It is obvious that the latency of the responses to attraction of the ear drum is much shorter than that of the responses to repulsion of the ear drum. Units with different characteristic frequencies have been similarly examined. In Fig. 5, the latencies of responses to magnetic driving of the ear drum and those of responses to click stimulation were plotted together on the same frequency time scale. The large solid circles represent the latencies of responses to clicks. The units with high characteristic frequencies have a shorter latency, while the units with low characteristic frequencies have a long latency. There is no linear relationship between the latencies of responses to clicks and their characteristic frequencies. The small solid circles labeled $\mathrm{A}$ and the empty circles labeled $\mathrm{R}$ indicate the latencies of the responses to magnetic driving of the ear drum outwardly and inwardly, respectively. The labels $A_{1}, A_{2}, A_{3}$ and $R_{1}, R_{2}, R_{3}$ represent the averaged delay time following attraction and repulsion, respectively, of the ear drum in the 


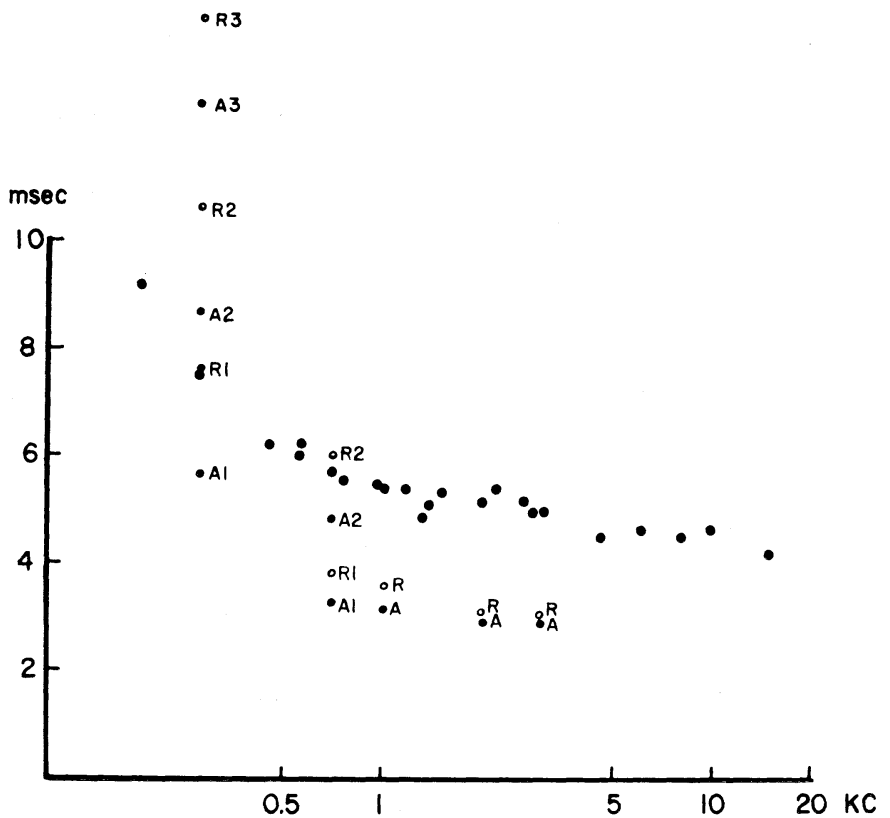

FIG. 5. Latencies of responses to electro-magnetic driving of the ear drum to either attraction (small solid circles: A, A1, A2, A3) or repulsion (small open circles: R, R1, R2, R3) and those of responses to click stimulation (large solid circles). The ordinate indicates the latency of responses in msec. and the abscissa the characteristic frequency.

units with low characteristic frequencies showing repetitive firing. The latency curve for click parallels that for magnetically driving the ear drum. This time difference corresponds to the velocity of sound in air from the speaker to the tested ear drum. The latencies of the responses to attraction of the ear drum are much shorter than those to repulsion in all of the units. Such a latency difference between attraction and repulsion of the ear drum is not constant but depends upon the characteristic frequency of the unit.

Exponential driving stimulus. The response pattern of a unit to slowly changing stimuli instead of rectangular stimuli is shown in FIG. 6 . In this case the rise time constant was $5 \mathrm{msec}$. From this series of experiment the author anticipated determining the level of trigger action for various stimulus time constants, but the relevant data is not available at present.

Responses to electro-magnetic driving of the ear drum during sound stimulation. Further evidence regarding the interaction between a step function stimulus of electro-magnetic driving of the ear drum and acoustic stimulation was obtained. In FIG. 7 the left upper record represents the responses to a 

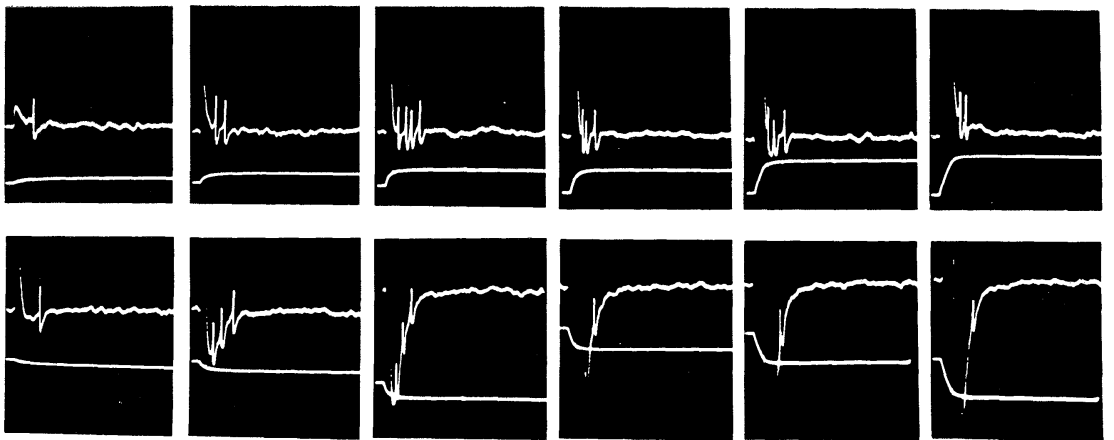

$30 \mathrm{~V}$

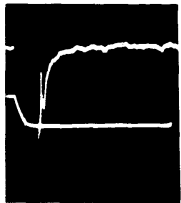

$40 \mathrm{~V}$

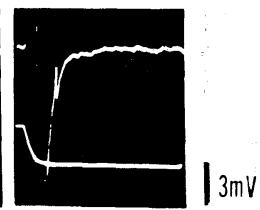

$50 \mathrm{~V} \overline{25 \mathrm{~ms}}$

upper : attraction

lower : repulsion

$\tau: 5 \mathrm{~ms}$

Fig. 6. Response pattern of a unit to exponential driving of the ear drum. Time constant in this case is $5 \mathrm{msec}$.

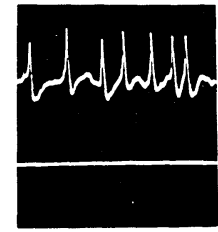

$400 \mathrm{cps}-40 \mathrm{~dB}$

tone

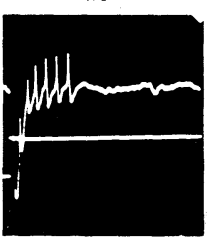

attraction

40 Volts

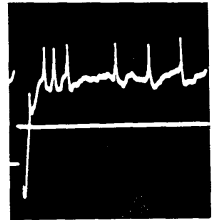

$-50 \mathrm{~dB}$

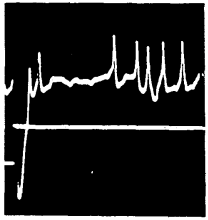

$-40 \mathrm{~dB}$

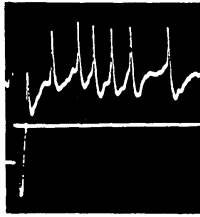

$-30 \mathrm{~dB}$

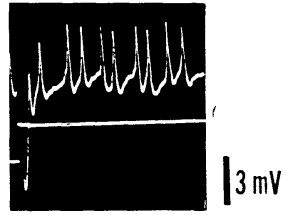

$-20 \mathrm{~dB} \overline{25 \mathrm{~ms}}$

FIG. 7. Responses to electro-magnetic driving of the ear drum during sound stimulation. See text.

$400 \mathrm{cps}$ tone at $-40 \mathrm{~dB}$ which is the characteristic frequency of this neuron and the left lower record represents the responses to attraction of the electromagnetically driven ear drum. Other records in FIG. 7 indicate the responses to the combined stimuli at different tone intensities having the same strength of attraction. As the intensity of sound was increased the burst activity was suppressed, and attraction of the ear drum likewise caused suppression of the responses to the tone. This latter phenomenon might account for the impaired 
hearing commonly occurring in humans when the ear drum is displaced under various atmospheric pressures.

\section{DISCUSSION}

The present experimental results indicate that the basilar membrane shift toward the scala vestibuli may have a triggering action. The responses of single primary auditory neurons to a step function driving of the ear drum occur only during attraction or repulsion and not during the steady phase of the stimulus. This indicates that the basilar membrane moves in response to the transient stimulus and produce a traveling wave along the basilar membrane according to its natural vibration. Each portion of the basilar membrane responds in a particular way to a momentary deflection of the ear drum. The units with high characteristic frequencies respond with only a single spike, while the units with low characteristic frequencies respond repetitively. As seen in FIG. 5, the latencies of spike responses to attraction are consistently shorter than those following repulsion of the ear drum. The latency difference between attraction and repulsion of the ear drum is not constant but depends upon the characteristic frequency of the unit; that is, for units with high characteristic frequencies the latency difference is small, but it is larger for the units with low characteristic frequencies. Consideration of this would appear important in understanding the mode of the vibration along the basilar membrane due to the sharp transient stimulus. BÉKÉsY, has reported that in the human ear a sudden change of sound pressure representing a rapidly declining transient of the ear drum produces a traveling wave along the basilar membrane. This wave at first progresses with great speed from the stapes toward the helicotrema but later moves more slowly. The latency curve for click stimulation in FIG. 5 is in agreement with this finding.

Considering the problem of directional sensitivity of the organ of corti, recently FLOCK, KIMURA, LUNDQUIST and WERSÄLL ${ }^{6)}$ have shown a consistent orientation of the sensory hairs which are organized in the shape of a W pointing toward the basal body. FLOCK and WERSÄLL ${ }^{7)}$ also reported that there are two groups of receptor cells in the lateral line organ of teleost fish, one consisting of cells polarized towards the head, the other consisting of cells polarized towards the tail.

From recordings of potentials from isolated nerve fibers from the lateral line organ of the ray, SAND ${ }^{9)}$ found that the frequency of the spontaneous activity in some nerve fibers increased when the canals were perfused in the head-tailward direction, and decreased in the other direction. In other fibers the reverse effects were observed. Later several author ${ }^{4,5,8,10)}$ confirmed this phenomenon in teleost and frog. Therefore, close relation may be highly pos- 
sible between such morphological and electrophysiological polarization of the hair cells in the cochlea and lateral line organ of fish.

\section{SUMMARY}

Responses of single primary auditory neurons to electro-magnetic driving of the ear drum were studied. From the latency measurement of the responses of neurons for either attraction or repulsion of the ear drum, trigger action may result from basilar membrane displacement toward the scala vestibuli. The traveling wave occurs along the basilar membrane when a step function type of stimulus is applied to the ear drum electromagnetically. This means that the basilar membrane moves due to its natural vibration for the transient effect and does not respond to the steady phase of the stimulus.

The author takes this opportunity of thanking Prof. Y. KATsuki for facilities to do this work. My thanks are also due to Dr. K. E. Chernetski, who kindly read the manuscript.

\section{REFERENCES}

1) Békésy, Georg von: On the resonance curve and the decay period at various points on the cochlear partition. J. Acous. Soc. Am. $21: 245-254,1949$.

2) Békésy, Georg von: Experiments in Hearing. McGraw-Hill Co. N. Y., 1960.

3) Davis, H., C. Fernandez, and D. R. McAuliffe: The excitatory process in the cochlea. Proc. Nat. Acad. Sciences. 36 : 580-587, 1950.

4) DijkgraAF, S.: Bau und Funktionen der Seitenorgane und des Ohrlabyrinths bei Fishen. Experientia. 8: 205-216, 1952.

5) DijkgraAF, S.: Elektrophysiologische Untersuchungen an der Seitenlinie von Xenopus laevis. Experientia. 12: 276-278, 1956.

6) Flock, Ake, R. Kimura, Per-Gotthard Lundquist, and Jan Wersäll: Morphological basis of directional sensitivity of the outer hair cells in the organ of corti. J. Acous. Soc. Am. 34 : 1351-1355, 1962.

7) Flock, Ake, ANd JAN Wersäll: Morphological Polarization and orientation of the hair cells in the labyrinth and the lateral line organ. J. cellular Biol. 15: 19-27, 1962.

8) Görner, P.: Beitrag zum Bau und zur Arbeitsweise des Seitenorgans von Xenopus laevis. Verh. dtsch. Zool. Ges. Saarbrücken. 193-198, 1961.

9) Sand, A.: The mechanism of the lateral sense organs of fishes. Proc. Roy. Soc. B. $123: 472-495,1937$.

10) Suckling, E. E. And Suckling, J. A.: The electrical response of the lateral line system of fish to tone and other stimuli. J. gen. Physiol. 34:1-18, 1950. 